\title{
Transformation from walls to disclination lines: Statics and dynamics of the pincement transition
}

\author{
Alberto de Lózar, ${ }^{*}$ Wolfgang Schöpf, ${ }^{\dagger}$ and Ingo Rehberg ${ }^{*}$ \\ Experimentalphysik V, Universität Bayreuth, D-95440 Bayreuth, Germany \\ Daniel Svenšek ${ }^{\S}$ \\ Department of Physics, University of Ljubljana, Jadranska 19, SI-1000 Ljubljana, Slovenia and Theoretische Physik III, Universität \\ Bayreuth, D-95440 Bayreuth, Germany \\ Lorenz Kramer \\ Theoretische Physik II, Universität Bayreuth, D-95440 Bayreuth, Germany
}

(Received 3 June 2005; published 17 November 2005)

\begin{abstract}
We present an experimental and theoretical study of the pincement phenomenon-transformation of a wall associated with the Fréedericksz transition into a pair of disclination lines. We measure the velocity of the boundary (front) between the two states as a function of the voltage. Experimental results are recovered by numerical simulations based on the nematic tensor order parameter, which also reveal the detailed threedimensional structure of the front. By introducing reduced models we obtain approximate expressions for the two-state coexistence voltage and the front velocity. We find a bifurcation scenario incorporating a pair of saddle nodes at which the wall and disclination solutions appear or disappear.
\end{abstract}

DOI: 10.1103/PhysRevE.72.051713

PACS number(s): 61.30.Eb, 61.30.Gd, 61.30.Jf, 61.30.Dk

\section{INTRODUCTION}

Defects not only are a typical and easily observable feature of liquid crystals, but also represent a bridge connecting liquid crystal studies to other areas of physics. The research of defects in liquid crystals has been particularly motivated by the daring idea of testing the cosmological Kibble mechanism [1] using a condensed matter system like ${ }^{4} \mathrm{He}$ quenched into the superfluid phase [2]. Later it was recognized that liquid crystals, nematics in particular, are experimentally much more convenient defect-forming systems [3]. Besides the cosmological context, this has also motivated studies of nematic defects per se, especially of their dynamics, which is certainly specific for the nematic liquid $[4,5]$ and cannot be applied to other condensed systems.

In a nematic liquid crystal, defects are of an orientational type and correspond to discontinuities of the nematic director. They are called disclinations; regarding their dimensionality they are classified into points and lines. While disclination lines are common and typical for nematics, point defects are encountered only in specially prepared confined geometries (e.g., the hedgehog and antihedgehog in the capillary [6]) due to their high distortion energy. In an electric or magnetic field also two-dimensional structures-walls-can exist, where, however, the director is not discontinuous but undergoes a continuous rotation when we pass through such a wall. Usually, walls are associated with the Fréedericksz transition in an electric or magnetic field, where they sepa-

\footnotetext{
*Electronic address: alberto.delozar@uni-bayreuth.de

${ }^{\dagger}$ Electronic address: wolfgang.schoepf@uni-bayreuth.de

*Electronic address: ingo.rehberg@uni-bayreuth.de

${ }^{\S}$ Electronic address: daniel.svensek@fmf.uni-lj.si

"Deceased.
}

rate field-aligned domains of opposite but equivalent director orientations. The statics and dynamics of Fréedericksz walls was studied in the 1970s by Léger, Brochard, and de Jeu et al. [7-10]. Theoretical and experimental studies [11,12] of disclinations are more recent as their description usually requires the tensor order parameter $[5,13,14]$.

The structural transition from a Fréedericksz wall into a pair of $\pm 1 / 2$ disclination lines - pincement, see Fig. 1-was first observed by Meyer [15] and was studied in the 1970s by Stieb et al. [16]. This transition has been recently reported by other groups $[17,18]$ in different geometries, but so far no systematic study has been performed.

In the present work we focus on the coexistence of the wall and disclination states and study the dynamics of the boundary (front) between these states. These structures can be visualized using polarizing microscopy as is shown in Fig. 2, where the wall and the two disclination lines may be distinguished. The front is the region that separates both structures. Experimental results will be compared to the results of direct numerical simulations based on the nematic tensor order parameter. Numerically, we also examine the detailed structure of the front, which by topological considerations contains a twist disclination line. We recover the main static and dynamic features by introducing a model of both states and the dissipation. We also propose a generic bifurcation model, explaining the observed nonlinear behavior of the front velocity at small voltages. (a)

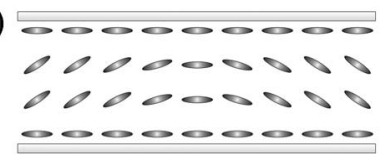

\section{(b)}

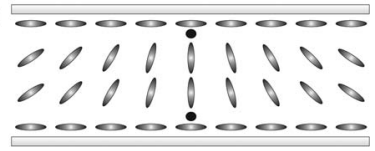

FIG. 1. Schematic director field presentation of the two possible transition regions between two equivalent Fréedericksz states: (a) domain wall and (b) a pair of disclination lines (indicated by the dots). 


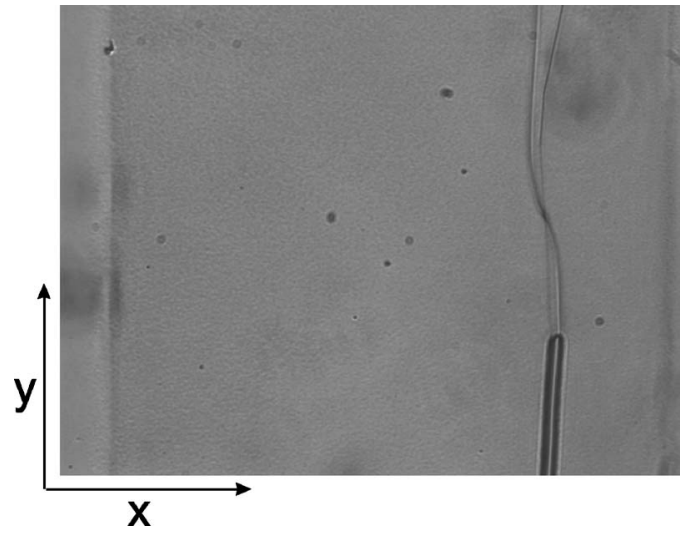

FIG. 2. Two disclination lines (upper part) in coexistence with the domain wall state (lower part) at $f=300 \mathrm{~Hz}$ and $U_{r m s}$ $=10.19 \mathrm{~V}$. The front between the two states moves upward with a velocity $v=13.40 \mu \mathrm{m} / \mathrm{s}$. One border of the channel (see Sec. II A) can be seen in the left part of the picture. The height and width of the image are 0.42 and $0.55 \mathrm{~mm}$, respectively.

\section{EXPERIMENT}

\section{A. Setup and procedure}

We used the standard experimental setup as described in Ref. [19]. The liquid crystal was placed between two parallel transparent electrodes [glass plates with an indium tin oxide (ITO) layer on one side]. The ITO surfaces were coated with a polymer and rubbed in one direction to produce planar alignment. The rubbing direction defines the $x$ axis, with the $y$ axis being perpendicular to $x$ in the layer plane. The thickness of the probe $d=18.8 \pm 1.0 \mu \mathrm{m}$ was determined using interferometry before the cell was filled. A detailed description of the sample preparation is presented elsewhere [20]. One of the electrodes has been treated by an etching process so that only narrow channels of ITO remain along the $y$ direction. For our experiments, we used a channel with a width of $0.6 \mathrm{~mm}$. This geometry introduces a slight inhomogeneity of the electric field which, due to our large width-toheight ratio of 20 , is confined to the vicinity of the channel boundaries. The measurements always took place in the homogeneous (inner) region of the channel.

A sinusoidal ac voltage $U(t)=\sqrt{2} U_{r m s} \cos (2 \pi f t)$ was applied across the electrodes by means of a wave form generator. We observed the appearing patterns under a polarizing microscope and recorded them with a charge-coupled device (CCD) camera connected to a frame grabber card. The images have a physical size of $552 \times 417 \mu \mathrm{m}^{2}$ and were digitized with a spatial resolution of $640 \times 484$ pixels into 256 gray scales at a rate of 60 images per second. The Fréedericksz domain wall and the disclination lines were visualized using the shadowgraph method [21]. Here the main feature is the transformation of a spatially modulated refractive index caused by the deflection of the director angle into quantitative light intensity information resulting from geometrical optics. The Fréedericksz transition itself was observed using the birefringence technique, where the probe is placed between two crossed polarizers both of which define an angle of $45^{\circ}$ with respect to the preferred director orientation $(x$ axis) $[22,23]$. The hydrodynamic flow produced by the CarrHelfrich mechanism [24-26] was avoided by working at a sufficiently high frequency.

We used a mixture of two standard liquid crystals 4-cyano-4'- $n$-pentylbiphenyl (5CB) and $N$-( $p$-methoxybenzylidene)-p-butylaniline (MBBA), which both exhibit the nematic phase at room temperature. Their physical properties are well documented [27-30]. The strong difference in the dielectric anisotropies of these liquid crystals $\left(\varepsilon_{a}=-0.53\right.$ for MBBA and 11.8 for $\left.5 \mathrm{CB}\right)$ allows for a mixture with an only slightly positive dielectric anisotropy by using a small quantity of $5 \mathrm{CB}$. Due to the small $\varepsilon_{a}$ of the mixture, the Fréedericksz transition occurs at a much higher voltage than for pure 5CB and thus a higher precision can be achieved. The liquid crystal was prepared by mixing $5 \mathrm{CB}$ and MBBA in a vial, using a mass concentration of $2.70 \%$ of $5 \mathrm{CB}$. Since the other parameters of MBBA and $5 \mathrm{CB}$ do not differ too strongly, we estimate all parameters of the mixture except $\varepsilon_{a}$ by the ones for MBBA. The dielectric anisotropy $\varepsilon_{a}=0.17$ was determined from the measurement of the splay Féedericksz threshold $U_{F}=\pi \sqrt{K_{11} / \varepsilon_{0} \varepsilon_{a}}=6.58 \mathrm{~V}$, where $K_{11}$ is the splay elastic constant. ${ }^{1}$ Two more mixtures were also prepared for further experiments. These mixtures have $\varepsilon_{a 1}$ $=0.09$ and $\varepsilon_{a 2}=0.31$, and were used in cells of thicknesses $d_{1}=22.0 \pm 1.0 \mu \mathrm{m}$ and $d_{2}=27.2 \pm 1.0 \mu \mathrm{m}$, respectively. All our measurements were performed at a temperature of $22{ }^{\circ} \mathrm{C}$ with a long-term stability of $\pm 5 \mathrm{mK}$.

The velocity of the front where the transformation of the Fréedericksz wall into a pair of disclination lines takes place was determined as follows. We first induced a Fréedericksz state in one of the narrow channels. Due to the inhomogeneity of the electric field at the borders of the channel a single domain wall appeared roughly in the middle of the channel. We then strongly increased the applied voltage $U_{r m s}$ to about $60 \mathrm{~V}$ so that the wall was spontaneously transformed into the disclination lines, which happened at some irregularity in the wall, typically at one end. We then decreased the voltage to the desired value. A typical picture of the front separating the two states is shown in Fig. 2. After waiting for about $20 \mathrm{~s}$ for the system to relax, we started measuring the velocity. Subsequently, further measurements at different voltages were performed. The whole procedure of inducing the Fréedericksz state and creating the front was repeated several times to take the data. The evolution of the front was followed with the CCD camera. After the system had relaxed, the camera started to sample images at a rate of 60 full frames per second for $5 \mathrm{~s}$. Our first goal was to extract the position of the transition between the disclination lines and the domain wall for each image.

In the two-dimensional intensity function of the image $I(x, y)$, the domain wall and the disclination lines present a strong contrast compared to the rest of the image. When walls more or less parallel to the $y$ direction are analyzed, this contrast leads to a strong variation within one horizontal line $I_{y}(x)$. Therefore, we first transformed the line $I_{y}(x)$ into

\footnotetext{
${ }^{1}$ The splay constants are very similar for both materials. At $25^{\circ} \mathrm{C}$, $K_{11}^{5 \mathrm{CB}}=6.5 \times 10^{-12} \mathrm{~N}$ and $K_{11}^{\mathrm{MBBA}}=6.66 \times 10^{-12} \mathrm{~N}$.
} 


$$
J_{y}(x)=\frac{1}{L+1} \sum_{i=-L}^{i=L}\left|\frac{I_{y}(x+i)}{\bar{I}}-1\right|
$$

where $\bar{I}$ is the mean intensity of the image. This simplifies the complex intensity variation corresponding to the wall and disclination lines by replacing it with a single peak. At the same time it averages the noise over $2 L+1$ pixels, with $L$ $=3$ in our case. The well defined maximum of $J_{y}(x)$ indicates the $x$ position of the wall or defect. Since the contrast of the wall is bigger than the contrast of the disclination lines, the maximum value of $J_{y}(x)$ can be an indicator of the defect type. Therefore, we defined two $y$-dependent functions: the value $J_{m}(y)$ and the position $x(y)$ of the maximum of $J_{y}(x)$. Changing $y$, the function $x(y)$ follows the wall with contrast $J_{m}(y)$ and is roughly constant up to the point $y_{\text {front }}$ where the wall is transformed into the two disclination lines. At this point, $x(y)$ follows one or both disclination lines (when they are not well separated) and $J_{m}(y)$ falls to a lower contrast. Therefore, by fitting to the function

$$
J_{m}(y)=\frac{1}{2}\left[J_{\text {wall }}+J_{\text {disc }}+\left(J_{\text {disc }}-J_{\text {wall }}\right) \tanh \left(y-y_{\text {front }}\right)\right]
$$

we obtained the $y$ coordinate of the interphase between both states as $y_{\text {front }}(t)$. The $x$ coordinate $x_{\text {front }}(t)$ was determined from the function $x(y)$ describing the position of the defects.

Linear fits provided the velocity $v=\sqrt{v_{x}^{2}+v_{y}^{2}}$ of the front with an accuracy better than $1 \%$. This procedure did not work when the patterns exhibited a very weak contrast. In these few cases the velocity was always very fast and good precision could be achieved performing the measurement by eye. After taking one set of data, we changed the applied voltage and began with a new acquisition, which we repeated several times to obtain the dependence of the front velocity on the voltage.

\section{B. Experimental results}

The front velocity dependence on the applied voltage is presented in Fig. 3. Positive velocities stand for the wall transforming into the disclination lines and negative velocities for the inverse process. For voltages lower than the Fréedericksz threshold $U_{F}=6.58 \mathrm{~V}$, the system is in the trivial planar configuration. For voltages between $U_{F}$ and $U_{\min }$ $=7.88 \mathrm{~V}$ the structure with the two disclination lines does not yet exist. If the voltage is reduced from a value above $U_{\text {min }}$, where this configuration is metastable, to a value below $U_{\min }$, the lines collapse and form the wall. In this case the velocity of the front is not defined. The first point of the plot $U_{\min }$ indicates the lowest voltage at which the disclination state is metastable. Above this voltage the transformation wall-disclinations takes place only in the interphase (front) between both states. A nonlinear dependence of the velocity on the voltage can be observed near $U_{\min }$, which we will discuss in Sec. IV D. At $U_{e q}=18.85 \mathrm{~V}$ the front is at rest, indicating that the configurations on either side of it have equal free energies. Thus, this is the coexistence point of the two states. If $U>U_{e q}$, the front moves toward the wall structure, and vice versa for $U<U_{e q}$. The velocity of the front is

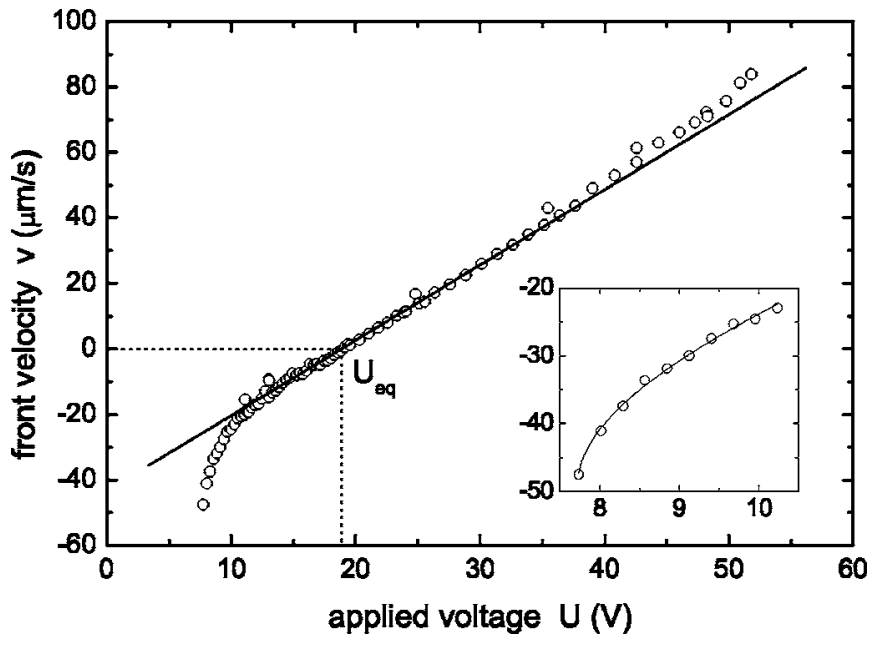

FIG. 3. Dependence of the front velocity on the voltage for a mixture with $\varepsilon_{a}=0.17$. Positive velocities represent an advancing disclination state. The velocity is zero at $U_{e q}=18.85 \mathrm{~V}$. The slope of the linear part at $U_{e q}$ is $k=2.30 \mu \mathrm{m} / \mathrm{s} \mathrm{V}$. Note that the velocity is curving up slightly with increasing voltage. Inset: fit with the prediction of the saddle-node model (32) of Sec. IV D.

proportional to the difference in free energies per unit length of the two structures, which represents a driving force of the front motion. In first order (for voltages close to $U_{e q}$ ) one expects a simple linear dependence $v=k\left(U-U_{e q}\right)$ for the front velocity $v$. Moreover, as hinted by the model equation (26) of Sec. IV C, the linear relationship should be valid over a reasonably broad voltage range, since the free energy difference of the two structures is, apart from a logarithmic correction, proportional to the voltage, while the dissipation is only a logarithmic function of the voltage. We fit a straight line to the measured velocities in an interval around $U_{e q}$, including 20 points above and below $U_{e q}$. The slope of the line is $k=2.30 \mu \mathrm{m} / \mathrm{s} \mathrm{V}$. The velocity is curving up slightly with increasing voltage.

Additional measurements of the equilibrium voltage $U_{e q}$ were performed with mixtures with different $\varepsilon_{a}$ (see Fig. 4). A linear dependence on $1 / \sqrt{\varepsilon_{a}}$ is observed, which indicates that $\varepsilon_{a}$ is sufficiently small so that the electric field is not distorted, i.e., $E=U / d[15]$.

\section{Twisted Fréedericksz wall}

Just above the Fréedericksz threshold the Fréedericksz wall is planar, i.e., it is a splay-bend wall. Due to the nematic elastic anisotropy, a secondary threshold exists at a higher voltage, leading to a twisted wall structure (Fig. 5) which is energetically less costly. The twist of the director may occur in two opposite directions, and hence two equivalent configurations are possible, giving rise to kinks in the Fréedericksz wall (Fig. 6). The straight section of the wall between adjacent kinks is a domain of the same twist direction. The twist transition threshold can be easily determined in both the experiment and simulation and thus represents a good test of the elastic anisotropy data.

When a ray of light passes through a liquid crystal, it is divided into the "ordinary" and "extraordinary" rays depend- 


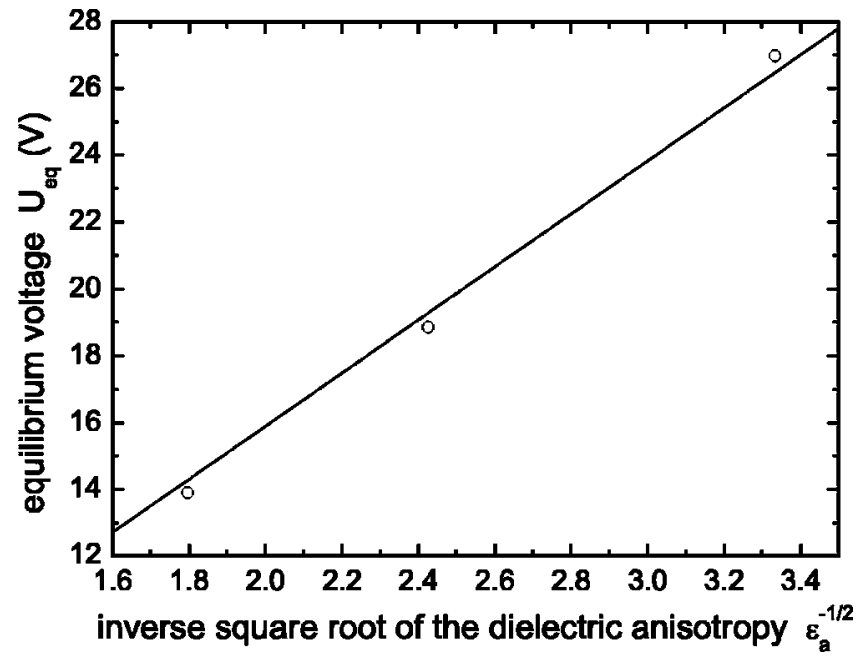

FIG. 4. Measured dependence of the equilibrium voltage $U_{e q}$ on the inverse square root of the dielectric anisotropy for three different mixtures (circles). The experimental points are fit by the line with a slope $d U_{e q} / d\left(\varepsilon_{a}^{-1 / 2}\right)=7.94 \mathrm{~V}$, which is forced to go through the origin.

ing on the polarization. This is accounted for by assigning to the liquid crystal two different indices of refraction $n_{\|}$and $n_{\perp}$ associated with the directions parallel and perpendicular to the director, respectively. In a pure splay-bend wall, the director lies in the $x z$ plane [see Fig. 1(a)]. The ray polarized in this plane is extraordinary, whereas the ordinary ray is polarized in the $y$ direction. Consequently, the extraordinary ray can be avoided by illuminating the sample with light polarized in the $y$ direction. In the planar wall configuration, this light keeps its polarization in the $y$ direction when passing the sample. An analyzer perpendicular to the polarizer is placed over the cell, so that the whole system is opaque. On the other hand, in the case of the twisted wall the polarization is no longer perpendicular to the director and is modified. Thus, the light that has passed through the cell has a nonzero component of the polarization parallel to the analyzer and hence it is transmitted. When observed under the microscope, two parallel, kinked (zigzag) lines are observed (Fig. 6). By rotating the analyzer, different colors show that the zig and zag regions between the kinks correspond to one of the two possible twist directions. This pattern is similar to the one observed by Chevallard et al. $[17,31,32]$ for a twisted wall in a homeotropic configuration subject to orthogonal magnetic and electric fields. There the angle between the zigs and the zags is typically around $90^{\circ}$, whereas in our case it is small.

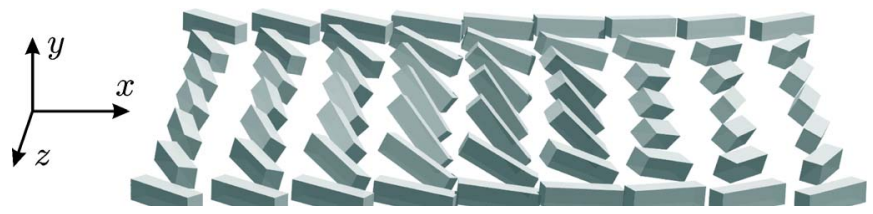

FIG. 5. (Color online) Simulated twisted Fréedericksz wall in a schematic presentation. Long axes of the boxes correspond to the director. The two opposite Fréedericksz states continue to the left and right, respectively.

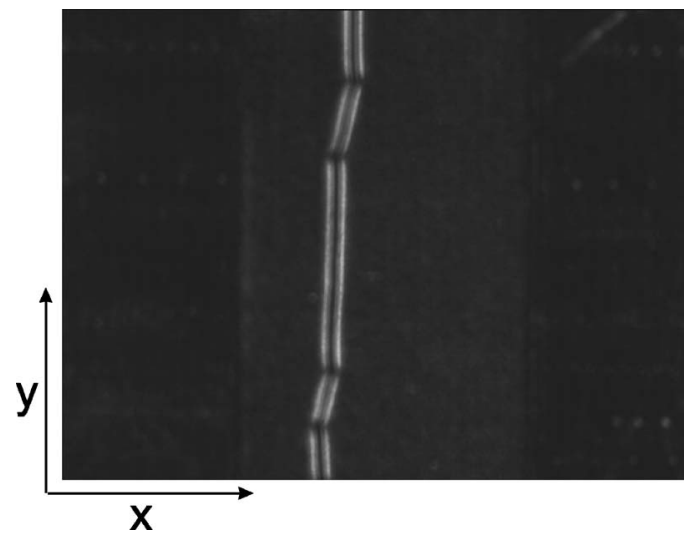

FIG. 6. Twisted Fréederikcsz wall observed between crossed polarizers. The zig and zag regions correspond to the two possible twist directions. The height and width of the image are 1.19 and $1.58 \mathrm{~mm}$, respectively.

The onset of the twist threshold was measured for the mixture with $\varepsilon_{a}=0.17$ in a cell with $d=90 \pm 5 \mu \mathrm{m}$. In the strong anchoring regime the threshold voltage is not expected to depend on the cell thickness. We started with the planar configuration increasing the voltage in small steps. After each voltage step, we waited $20 \mathrm{~s}$ for the system to relax before the transmitted intensity was taken. This waiting time is about two orders of magnitude longer than the relevant director relaxation time $\tau_{d} \sim \gamma_{1} \xi_{E}^{2} / K_{a} \sim 0.2 \mathrm{~s}$, where $\gamma_{1}$ is the rotational viscosity, $\xi_{E}$ the electric coherence length defined in Sec. IV A, and $K_{a}$ the elastic anisotropy between splay-bend and twist, suitably defined as $K_{a} \sim\left(K_{11}+K_{33}\right) / 2$ $-K_{22}$.

In Fig. 7 the resulting curve is shown. First a smooth change in the intensity is observed due to the variations in the interference between the ordinary and extraordinary rays, which is typically seen with the birefringence technique [23]. Here the interference can be observed because the light was not completely blocked by the analyzer. A strong change in the curve determines the threshold voltage $U_{\text {twist }}=13.08 \mathrm{~V}$. The ratio $U_{\text {twist }} / U_{F}=1.99$ agrees perfectly with the value ob-

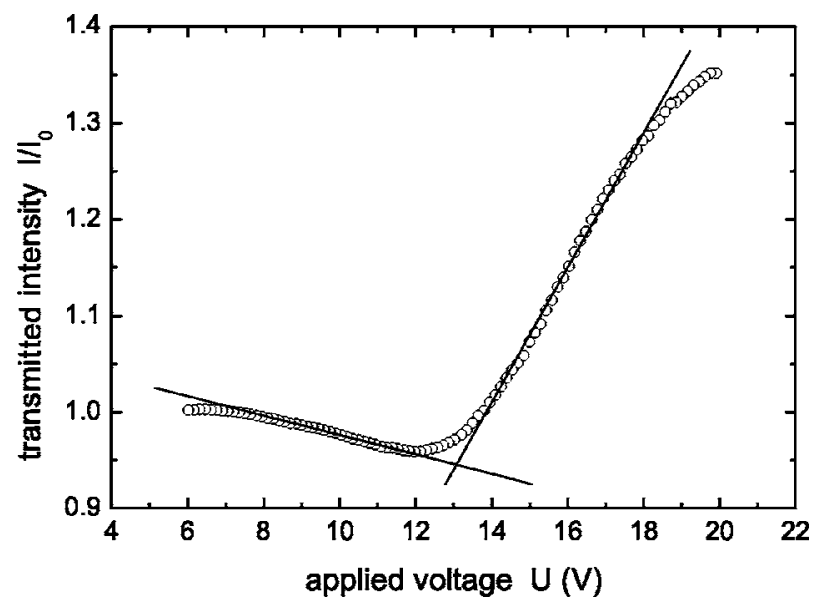

FIG. 7. Detection of the twisted Fréedericksz wall. A strong change of the transmitted intensity determines the threshold voltage at $U_{\text {twist }}=13.08 \mathrm{~V}$. 
tained in the simulation, which is $\left(U_{t w i s t} / U_{F}\right)_{s i m}=2.0$. This signifies that the elastic anisotropy assumed in the simulations corresponds to the actual one.

\section{NUMERICAL SIMULATION}

\section{A. Equations and method}

The experimental results are to be compared to the results of a direct numerical simulation. Due to the presence of defects, the complete nematic tensor order parameter $Q$ must be considered in the simulation as demonstrated for example in Ref. [14]. Here we briefly present the equations and the method of solving them to obtain the time evolution of the $\mathrm{Q}$ tensor field. In the uniaxial case, the $Q$ tensor is usually expressed as $\mathbf{Q}_{i j}=S\left(3 n_{i} n_{j}-\delta_{i j}\right) / 2$, where $\mathbf{n}$ is the director and $S$ the degree of order. We keep the $\mathrm{Q}$ tensor general; the uniaxial limit presented should serve only as a definition of $S$. The free energy density consists of homogeneous, distortion (elastic), and dielectric parts, $f(\mathbf{Q}, \nabla \mathbf{Q})=f_{h}+f_{d}+f_{e}$, reading

$$
\begin{gathered}
f_{h}=\frac{1}{2} A Q_{i j} \mathrm{Q}_{j i}+\frac{1}{3} B \mathrm{Q}_{i j} \mathrm{Q}_{j k} \mathrm{Q}_{k i}+\frac{1}{4} C\left(\mathrm{Q}_{i j} \mathrm{Q}_{j i}\right)^{2} \\
f_{d}=\frac{1}{2} L\left(\partial_{i} \mathrm{Q}_{j k}\right)^{2}+\frac{1}{2} L^{\prime}\left(\partial_{i} \mathrm{Q}_{i k}\right)\left(\partial_{j} \mathrm{Q}_{j k}\right)+\frac{1}{2} L^{\prime \prime} \mathrm{Q}_{i j}\left(\partial_{i} \mathrm{Q}_{k l}\right)\left(\partial_{j} \mathrm{Q}_{k l}\right),
\end{gathered}
$$

$$
f_{e}=-\frac{1}{3} \varepsilon_{0} \varepsilon_{a m} Q_{i j} E_{i} E_{j},
$$

where $A=a\left(T-T^{*}\right), B$, and $C$ are the Landau coefficients describing the nematic-isotropic phase transition, $T$ and $T^{*}$ are the temperature and supercooling temperature, $L, L^{\prime}, L^{\prime \prime}$ are the elastic constants, $\mathbf{E}$ is the electric field, and $\varepsilon_{a m}$ is the microscopic dielectric anisotropy giving rise to the usual (macroscopic) anisotropy $\varepsilon_{a}=S \varepsilon_{a m}$. As confirmed by Fig. 4, we can ignore the distortion of the electric field due to director deformation and assume the electric field in the sample is $\mathbf{E}=(U / d) \hat{\mathbf{e}}_{z}$, normal to the substrates. The distortion part $f_{d}$ is represented by the minimum set of terms to distinguish between splay, twist, and bend distortions:

$$
\begin{gathered}
K_{11}=\frac{9 S^{2}}{4}\left(2 L+L^{\prime}-S L^{\prime \prime}\right), \\
K_{22}=\frac{9 S^{2}}{4}\left(2 L-S L^{\prime \prime}\right), \\
K_{33}=\frac{9 S^{2}}{4}\left(2 L+L^{\prime}+S L^{\prime \prime}\right),
\end{gathered}
$$

where $K_{11}, K_{22}$, and $K_{33}$ are the Frank elastic constants for splay, twist, and bend, respectively. Note that a term cubic in $\mathrm{Q}$ is required to distinguish between splay and bend distortions. With Eqs. (6)-(8) and for constant $S$, the distortion part (4) reduces to the standard splay, twist, and bend Frank elastic terms [15], while Eq. (5) reduces to the standard dielectric term [15]. In the uniaxial case the homogeneous contribution (3) is a function of $S$ only and reduces to

$$
f_{h}(S) \equiv \frac{3}{4} A S^{2}+\frac{1}{4} B S^{3}+\frac{9}{16} C S^{4} .
$$

The free energy of the system is

$$
F=\int d V\left(f-\lambda Q_{i i}-\lambda_{i} \epsilon_{i j k} Q_{j k}\right),
$$

where the Lagrange multipliers appear due to the requirement that $Q$ be traceless and symmetric and have no physical meaning. Neglecting hydrodynamic flow, the order parameter evolves according to a time-dependent Ginzburg-Landau equation:

$$
\mu_{1} \frac{\partial \mathbf{Q}_{i j}}{\partial t}=\nabla \cdot \frac{\partial f}{\partial\left(\nabla Q_{i j}\right)}-\frac{\partial f}{\partial Q_{i j}}+\lambda \delta_{i j}+\lambda_{k} \epsilon_{k i j},
$$

where $\mu_{1}$ is the tensorial analog of the director rotational viscosity $\gamma_{1}=S^{2} \mu_{1}$ [33]. The Lagrange-multiplier terms state that the isotropic and antisymmetric components are not specified by Eq. (11) and are determined by the constraints. One gets rid of them by subtracting from Eq. (11) its isotropic and antisymmetric parts:

$$
\mu_{1} \frac{\partial \mathbf{Q}_{i j}}{\partial t}=\left\langle\nabla \cdot \frac{\partial f}{\partial\left(\nabla Q_{i j}\right)}-\frac{\partial f}{\partial \mathbf{Q}_{i j}}\right\rangle,
$$

where \langle\rangle denotes the traceless symmetric part.

Equation (12) is discretized on a two- or threedimensional rectangular grid and solved explicitly in time. At every time step one calculates the right-hand side of Eq. (12), takes its traceless symmetric projection, and updates the $\mathrm{Q}$ field. The uniaxial ansatz for $\mathrm{Q}$ with $S=S_{0}$ is used as the initial condition, where $S_{0}$ is the bulk degree of order. The boundary condition corresponds to strong anchoring: at the substrates the director is fixed in the $x$ direction, while at the lateral boundaries (with normals along $x$ and $y$ ) we use the von Neumann condition. To obtain the wall structure we start with an appropriately perturbed homogeneous director field, whereas for the disclination structure the director field of a disclination pair in its equilibrium configuration is implemented initially.

The tensor description introduces a microscopic length scale-the nematic correlation length $\xi_{S}$, suitably defined as

$$
\xi_{S}=\sqrt{\frac{3}{2} \frac{L}{f_{h}^{\prime \prime}\left(S_{0}\right)}} .
$$

A typical value for the correlation length is $\xi_{S} \sim 6 \mathrm{~nm}$ and a corresponding characteristic time scale is $\tau=\mu_{1} \xi_{S}^{2} / L$ $\sim 0.5 \mu \mathrm{s}$. This sets severe limits to length and time scales one can achieve in the simulations, but a better alternative is not known so far. The simulated systems are typically two or three orders of magnitude smaller than the experimental ones.

The correlation length $\xi_{S}$ cannot be measured in the experiment. It does, however, necessarily enter the numerical calculations when defects are present. We use the Landau constants from Ref. [34] and adjust the temperature (the value of $A$ ) such that the degree of order $S$ in our simulations agrees with that found in MBBA [35] at the temperature of the experiment. The tensorial elastic constants are then calculated from known Frank elastic constants of MBBA by use of Eqs. (6)-(8). Consequently, the value of the correlation 


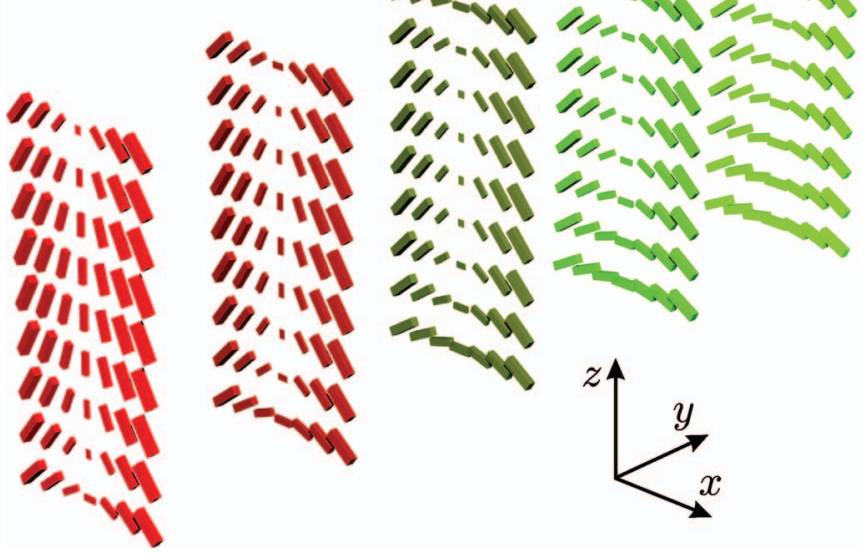

FIG. 8. (Color) Middle part of the cross section through the front: the defect structure (left) transforms to the wall (right) via a $1 / 2$ twist disclination line (middle) normal to the substrates. The nematic $Q$ tensor field is represented by the boxes; the lengths of the edges correspond to the eigenvalues (a constant is added to make them nonnegative). The distance between the $x z$ slices has been increased for clarity; in reality it is comparable to the distance between the boxes in the slice.

length is only approximate, which implies that the length scale of the simulation is not precisely known. Note, however, that the observable quantities depend only weakly (through the logarithm) on the value of the correlation length, as suggested by the models (18) and (26) of Sec. IV. Taking an alternate point of view, the measurement of the pincement threshold voltage, which depends on the ratio $d / \xi_{S}$, could eventually serve as an indirect probe of the correlation length.

\section{B. Structure of the front}

The front possesses a submicrometer-range structure and thus it cannot be resolved in the experiment. The simulation, however, suggests a rather nontrivial configuration (Figs. 8 and 9). The Fréedericksz wall is transformed into two disclination lines via a twist disclination line with winding number $1 / 2$, oriented perpendicular to the substrates and connecting the two disclination lines. When we encircle the twist disclination line, the director undergoes a $\pi$ rotation about the $y$ axis; this rotation corresponds to the twist deformation (Fig. 9). For comparison, in the case of the wedge disclination line (the disclination lines of the defect state are of the wedge type), which is somehow easier to visualize, the director rotates about the axis parallel to the line. The tensorial structures of the twist and wedge disclinations are qualitatively equivalent, i.e., the profiles of the degree of order and biaxiality are the same, only the tensorial axes are interchanged. It should be pointed out that the front must contain the (twist) disclination line for topological reasons, i.e., its existence is not in any way subject to the tensorial description used. The latter merely allows us to calculate its structure without having to introduce a microscopic cutoff.

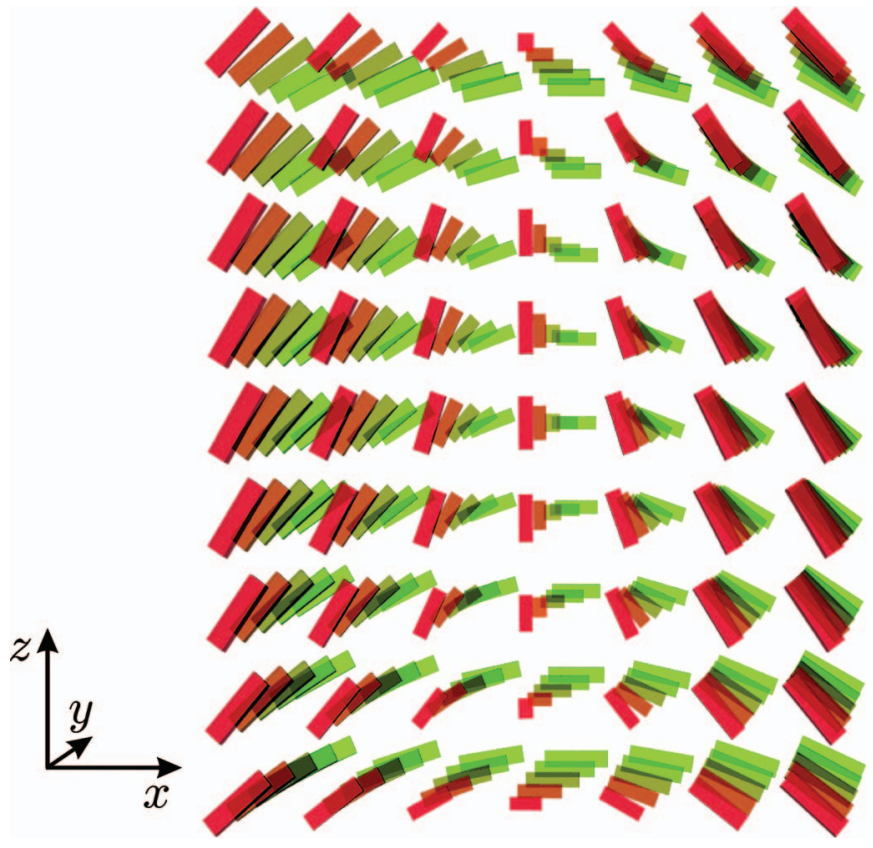

FIG. 9. (Color) A view through the front (from left to right in Fig. 8), revealing the $\pi$ twist rotation around the $1 / 2$ twist disclination line in the middle. Note that it is required topologically since the director in the center of the Fréedericksz wall points in the $x$ direction, whereas the central director of the disclination configuration is parallel to $z$. The color coding is consistent with the one in Fig. 8 and helps to distinguish between near (red) and distant (green) regions.

\section{Coexistence and front motion}

The equilibrium voltage was determined by computing the free energies of the wall and defect configurations as functions of the voltage and then finding the voltage where they are equal. We could simulate cells with thicknesses up to $2 \mu \mathrm{m}$ and then extrapolated the result (Fig. 10) to thicker cells using the estimate (18) of Sec. IV A. Figure 10 indicates that the model is valid. The deviation of $12 \%$ can be attributed to the uncertainty in the length scale of the simu-

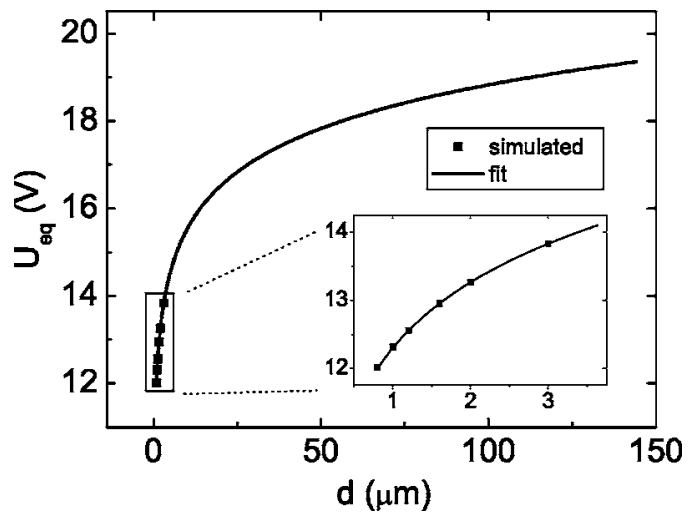

FIG. 10. Numerically determined pincement threshold voltage $U_{e q}$ vs cell thickness $d$, fitted using the estimate (18). For $d$ $=19 \mu \mathrm{m}$ we get $U_{e q}=16.5 \mathrm{~V}$; the experimental value is $U_{e q}$ $=18.85 \mathrm{~V}$. 


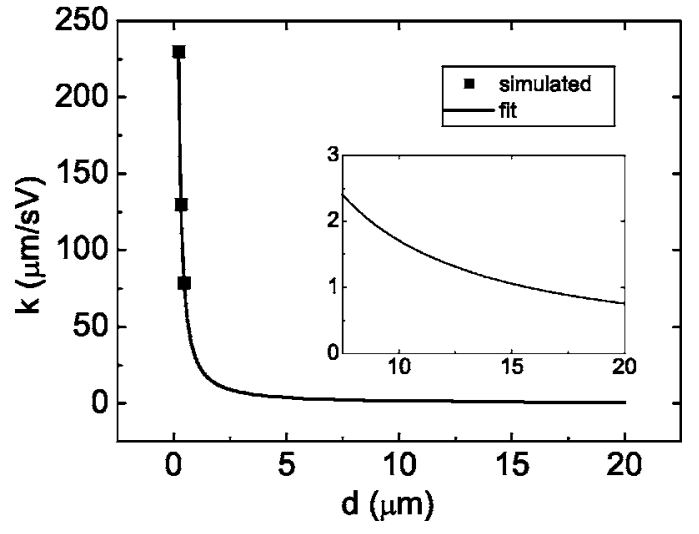

FIG. 11. The front velocity-voltage coefficient, $v=k\left(U-U_{e q}\right)$, vs cell thickness, fitted by the model (28). The inset shows this function magnified in the region of experimentally relevant thicknesses; at $d=19 \mu \mathrm{m}$ it reads $0.8 \mu \mathrm{m} / \mathrm{s} \mathrm{V}$.

lation, to a finite anchoring strength which effectively increases the cell thickness, and possibly also to ionic impurities screening the electric field in the cell.

A three-dimensional numerical computation is required to simulate the front motion. The simulation was started with the wall structure in one half of the computational domain and the defect structure in the other. Then the system was left to relax in the electric field. The front, which contains a $1 / 2$ twist disclination (Figs. 8 and 9), was quickly formed-on the time scale of $\tau$. The position of the front was detected by locating the core of the twist disclination. The velocity of the front was left to saturate to yield the $v(U)$ dependence. The sample had to be sufficiently large in order to overcome the transient and reach the saturation, i.e., the regime of constant velocity. We could simulate cells with thicknesses up to $0.46 \mu \mathrm{m}$ (Fig. 11).

The simulated front velocity in thin cells is much higher than the values measured in thick cells, but it is found to be a decreasing function of the thickness. The simulated cells are still too thin compared to the experimental ones and do not enable us to make a direct comparison of numerical and experimental data. Therefore we extrapolated the numerical data to thicker cells using a model [Eq. (28) of Sec. IV C] describing the dependence of the velocity on the thickness of the cell. According to this model, apart from logarithmic corrections the velocity is inversely proportional to the cell thickness. The fit is presented in Fig. 11; for $d=19 \mu \mathrm{m}$, it yields $k=0.8 \mu \mathrm{m} / \mathrm{s} \mathrm{V}$. The model (27) with no fitting parameters also gives $k=0.8 \mu \mathrm{m} / \mathrm{s} \mathrm{V}$. This value is three times smaller than the measured one, which hints that the dissipation might be overestimated in the model. The main source of error, however, appears to be the effective confinement of the system in the $x$ direction due to the limited number of computational points in the simulation. Another, presumably minor source of the discrepancy is the backflow effect which was neglected throughout this study.

\section{MODEL}

\section{A. Statics}

In this section we present a static model of the wall and the disclination structure, which yields the equilibrium volt- age, i.e., the coexistence point of the two states. We restrict ourselves to a two-dimensional description where the relevant effects can be incorporated at least qualitatively. The simplest description of elastic distortions in nematics is given by the one-elastic-constant director theory with the elastic and dielectric free energies

$$
F=\int d V\left(\frac{1}{2} K(\nabla \phi)^{2}-\frac{\varepsilon_{0} \varepsilon_{a}}{2} E^{2} \sin ^{2} \phi\right),
$$

where $\phi$ is the angle between the director lying in the $x z$ plane and the $x$ axis, $E$ is the electric field applied along $z, K$ is the elastic constant, and $\varepsilon_{a}$ is the dielectric anisotropy of the nematic phase. The electric field can be considered not distorted, $E=U / d$. The model (14) introduces a characteristic length scale-the electric coherence length $\xi_{E}=U_{0} / E$, where $U_{0}=\sqrt{K /\left(\varepsilon_{0} \varepsilon_{a}\right)}$ is a suitable voltage unit to be used in the following.

The planar Fréedericksz transition (also called the splay transition) takes place at the Fréedericksz threshold voltage $U_{F}=\pi U_{0}$, where the splay elastic constant must be used in $U_{0}$ in this case [15]. The distorted state in the nonlinear regime above the Fréedericksz transition can be expressed analytically in terms of elliptic functions [23]. One finds that $\phi$ reaches $\pi / 4$ at midplane for $U^{2} / U_{F}^{2} \approx 1.4$.

The Fréedericksz wall can be captured analytically either slightly above or well above $U_{F}$. In the first case the variational ansatz $\phi=\cos (\pi z / d) f(x)$ together with an expansion of the last term in Eq. (14) up to fourth order leads to a wall energy per unit length $F_{\text {wall }} / l=\alpha \varepsilon_{0} \varepsilon_{a} U_{0} U$ with $\alpha=(\sqrt{8} / 3)(1$ $\left.-U_{F}^{2} / U^{2}\right)^{3 / 2}$. In the other case the solution exhibits essentially no $z$ dependence except for boundary layers, which may be neglected in the free energy. Thus one is left with a one-dimensional problem: the director is parallel to the field on both sides of the wall with a $\pi$ turn in between. We then find $\alpha=\sqrt{2}$ and a wall half-width $\zeta_{e}=d\left(U_{0} / U\right)=\xi_{E}$.

Defects can be incorporated in this framework only as singular points where $\phi$ is not defined. In polar coordinates $(r, \varphi)$ an isolated disclination at the origin at zero field is given by $\phi= \pm \varphi / 2+\phi_{0}$ and its energy per unit length is

$$
F_{r_{c}} / l=\gamma \pi K \ln \left(\frac{r_{c}}{\xi}\right)=\gamma \pi \varepsilon_{0} \varepsilon_{a} U_{0}^{2} \ln \left(\frac{r_{c}}{\xi}\right),
$$

where $\gamma=1 / 4$ and upper and lower cutoffs have been introduced. Whereas the divergence of the energy with increasing system size is realistic, the lower cutoff $\xi$, which is very close to $\xi_{S}$ defined in Sec. III, disappears in theories incorporating the degree of order. There are two simple ways to incorporate perturbations of the director field of an isolated disclination into its free energy. One is to adjust the upper cutoff and the other is to include the effect of a nonuniform dependence of $\phi$ on the polar angle $\varphi$. Writing $\phi=f(\varphi)$ $+\phi_{0}$ with $f(2 \pi)=\pi$, one recovers the expression (15) with $\gamma=\left\langle f^{\prime}(\varphi)^{2}\right\rangle$, where $\langle\cdots\rangle$ denotes averaging over $\varphi$. A nonuniform distribution of $\phi$ will always increase the energy of the disclination. When the disclination is pushed all the way to a boundary $\phi$ rotates over its full range $\pi$ when $\varphi$ rotates over the half space $\pi$, and then $\gamma=1 / 2$. 
Let us estimate the voltage $U_{e q}$ where the energy of the Fréedericksz wall equals that of the pinched wall (i.e., the disclination structure). This voltage is well above the Fréedericksz threshold, so we should take

$$
F_{\text {wall }} / l=\sqrt{2} \varepsilon_{0} \varepsilon_{a} U_{0} U
$$

for the energy per unit length of the regular wall. For the pinched wall we just take the energy of two defects with upper cutoff $r_{c}=\xi_{E}$ since at larger distances the distortion due to the disclination is suppressed by the electric field:

$$
F_{d e f} / l=2 \gamma \pi \varepsilon_{0} \varepsilon_{a} U_{0}^{2} \ln \left(\frac{\xi_{E}}{\xi}\right) .
$$

The factor $\gamma$ should lie between the isolated disclination value $1 / 4$ and the half-space value $1 / 2$, because director rotation is effectively excluded in the central part of the pinched wall.

Equating the two energies gives

$$
\frac{U_{e q}}{U_{0}}=\sqrt{2} \gamma \pi \ln \left(\frac{d}{\xi} \frac{U_{0}}{U_{e q}}\right) .
$$

As $d \gg \xi$, the dependence of $U_{e q}$ on $d$ is roughly logarithmic. The accuracy of the estimate (18) is expected to improve as $d / \xi$ grows larger, as in this limit the energy associated with the details of the director distortion due to the electric field is small compared with the distortion energy of the defects. In the experiment we have $d / \xi \sim 3500$, which is large enough for the expression (18) to be credible. However, note that the energy of the defect is only a logarithmic function of its size. As presented in Fig. 4, in the experiment we measure $d U_{e q} / d\left(\varepsilon_{a}^{-1 / 2}\right)=7.94 \mathrm{~V}$. With this value, assuming $\xi=6 \mathrm{~nm}$ and using the splay constant as the elastic constant, Eq. (18) gives $\gamma \approx 1 / 2.4$.

\section{B. Bifurcation scenario}

In this section we want to explain the most important features of the bifurcation scenario associated with the pincement transition, which are presented in Fig. 12. Although the proper description of the degree of orientational order includes the tensor order parameter, here we use a somewhat simpler complex order parameter $\tilde{A}=|\tilde{A}| e^{\mathrm{i} \theta}$ with $\theta=2 \phi$, which is more convenient for analytic work. Note that thereby the symmetry $\phi \leftrightarrow \phi+\pi$ is still respected. We consider the simplest Ginzburg-Landau expression for the free energy

$$
F=\int d^{3} \widetilde{r}\left(\frac{1}{2} D|\nabla \widetilde{A}|^{2}+\frac{1}{2} \alpha|\widetilde{A}|^{2}+\frac{1}{4} \beta|\widetilde{A}|^{4}+\frac{1}{2} \widetilde{h}\left(\widetilde{A}+\tilde{A}^{*}\right)\right),
$$

where the last term incorporates the external field. Here the correlation length is given by $\xi_{0}=\sqrt{D / \alpha}$. In order to relate the other model parameters to the liquid crystal material parameters we consider a situation with small spatial gradients (on the scale $\xi_{0}$ ) and small external field. One then obtains $|\widetilde{A}|_{\infty}^{2}=\alpha / \beta$. With the relations

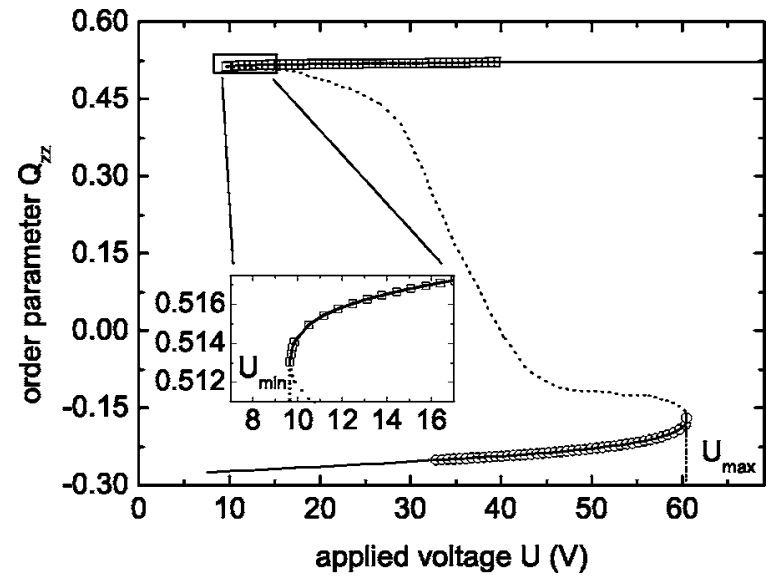

FIG. 12. A bifurcation diagram indicating the wall (circles) and disclination (squares) solutions and the unphysical unstable branch (dotted line). The data presented as circles and squares are calculated in the tensor simulation for a system with thickness $d$ $=0.64 \mu \mathrm{m}$, whereas the unstable branch connecting the two saddle nodes is added by hand and is only schematic. As a convenient order parameter we choose the $\mathrm{Q}_{z z}$ component of the nematic order parameter in the middle of the wall, measuring the ordering with respect to the $z$ axis (see Sec. IV D).

$$
4 D \frac{\alpha}{\beta}=K, \quad \tilde{h} \sqrt{\frac{\alpha}{\beta}}=\frac{1}{4} \varepsilon_{0} \varepsilon_{a} E^{2}
$$

one recovers Eq. (14) from Eq. (19). Introducing dimensionless quantities

$$
r=\widetilde{r} / \xi_{0}, \quad A=\sqrt{\frac{\beta}{\alpha}} \widetilde{A}, \quad h=\widetilde{h} \frac{\beta^{1 / 2}}{\alpha^{3 / 2}}=\varepsilon_{0} \varepsilon_{a} \frac{E^{2} \xi_{0}^{2}}{K},
$$

into the free energy (19) leads to the Ginzburg-Landau equation

$$
\tau \partial_{t} A=\Delta A+\left(1-|A|^{2}\right) A-h .
$$

Setting $A=u+\mathrm{i} v$, the stationary homogeneous solutions are given by $v_{0}=0$ and $\left(1-u_{0}^{2}\right) u_{0}=h$. For $h<\sqrt{4 / 27} \approx 0.385$ there are three solutions, which for $h \ll 1$ are $-1-h / 2,1$ $-h / 2$, and $h$. The one with the largest absolute value corresponds to $\mathbf{n} \| \mathbf{E}$ and the next smaller one to $\mathbf{n} \perp \mathbf{E}$. The condition $|A| \approx 1$, which requires $h \ll 1$, is the condition that (14) is valid.

When following the wall solution to (experimentally unreachable) high fields, one finds that it exists for $h_{F}<h$ $<h_{\max }$, i.e., $U_{F}<U<U_{\max }$ (see Fig. 12). For $h / h_{F}=U^{2} / U_{F}^{2}$ $\gg 1$ it is captured within the one-dimensional description introduced above and $h_{\max }$ is easily determined numerically giving $h_{\max }=0.0515$. At $h_{\max }$ the wall solution disappears in a saddle-node bifurcation (the stable wall solution merges with an unstable one) and the system jumps into the disclination state with the defect lines very near to the cell boundaries (in the one-dimensional geometry the wall vanishes completely). Physically, the defect pair would nucleate when the width of the wall is comparable to the nematic correlation length, i.e., 
$U_{\text {max }} \sim U_{0} d / \xi \sim 5 \mathrm{kV}$ which is, of course, never accessible in practice. In Fig. $12 U_{\max }$ is much lower due to the small cell thickness there.

The disclination state can be followed to lower fields (see Fig. 12) where it eventually disappears at $U_{\min }$ in another saddle-node bifurcation. There the two defects annihilate and a regular wall is reestablished. Actually $U_{\min }$ is only slightly larger than $U_{F}$. The experiment gives $U_{\min }^{2} / U_{F}^{2} \approx 1.4$, where $U_{F}=\pi U_{0}$. In the $\mathrm{Q}$ tensor simulation we find that $U_{\min }$ varies appreciably with the sample thickness. We get $U_{\min }^{2} /\left(\pi U_{0}\right)^{2}$ $\approx 5.21,4.75,4.53$ for $d=0.32,0.64,0.96 \mu \mathrm{m}$.

In the disclination state the defects are repelling and their position is fixed only by the boundaries; the voltage interval where their equilibrium position is not at the boundaries is extremely narrow. Hence, in an estimate the bifurcation at $U_{\text {min }}$ can be viewed as the point when the force on the two defects changes from a repelling to an attractive one (not considering the force due to the boundaries). For thick cells this force mainly comes from the electric field, whereas in thin cells also the defect interaction contributes, as hinted by the thickness dependence of $U_{\min }$ obtained numerically. Above and below the two defects one has the regular Fréedericksz wall where the director rotates through the planar orientation when crossing the wall. On the other hand, between the defects it rotates in the reverse sense through the homeotropic orientation $(\phi=\pi / 2)$. One has to consider the competition between these two wall types. Recall that in the Fréedericksz distorted state (far away from the wall) the director angle $\phi$ reaches a midplane value $\pi / 4$ when $U_{\min }^{2} /\left(\pi U_{0}\right)^{2} \approx 1.4$. If we disregard the elastic anisotropy, beyond this voltage it is thus elastically favorable for the director to rotate through the homeotropic orientation in the midplane region. Actually the ratio 1.4 should be an overestimate due to the dielectric energy and elastic anisotropy not taken into consideration. The increase of $U_{\min }^{2} /\left(\pi U_{0}\right)^{2}$ with decreasing thickness is a signature of the interaction between the defects, which effectively increases with decreasing thickness.

\section{Dynamics}

To describe the dynamics, i.e., the motion of the front, the model must be extended to include the dissipation taking place when the front is moving. The rate of the decrease of the free energy $\dot{F}=v \Delta F / l$, where $\Delta F$ is the free energy difference of the disclination and wall states obtained from Eqs. (16) and (17),

$$
\Delta F / l=\varepsilon_{0} \varepsilon_{a} U_{0}^{2}\left[2 \gamma \pi \ln \left(\frac{d}{\xi} \frac{U_{0}}{U}\right)-\sqrt{2} U / U_{0}\right],
$$

is equal to the dissipation. The front essentially consists of the $1 / 2$ twist disclination line of length $d$, contained in an effective cylinder of radius $\xi_{E}$ (Figs. 8 and 9). Outside of this cylinder, the distortion due to the disclination is suppressed by the electric field. Hence, the dissipation due to the moving front is essentially given by the one of the moving twist disclination line confined to the radius $\xi_{E}$. With the help of Fig. 9 one can perceive how the director in a point of space is rotated when the front (twist disclination) passes this point. In the one-constant approximation, the twist disclination is equivalent to the wedge one. Assuming the disclination is in the equilibrium configuration, the dissipation $\dot{F}$ is simply

$$
\dot{F}=-\gamma_{1} d \int_{0}^{2 \pi} d \varphi \int_{\xi}^{\xi_{E}} r d r \dot{\mathbf{n}}^{2},
$$

with $\dot{\mathbf{n}}=-\mathbf{v} \cdot \nabla \mathbf{n}$ and $\mathbf{v}$ the velocity of the disclination, which gives [36]

$$
\dot{F}=-\frac{\pi}{4} \gamma_{1} d v^{2} \ln \frac{\xi_{E}}{\xi} .
$$

Note that the upper cutoff in Eq. (25) appears already intrinsically due to the disclination motion [37] and is of the order of $K /\left(\gamma_{1} v\right)$, which compares to $\xi_{E}$ in our case.

From Eqs. (23) and (25) thus follows

$$
v=\frac{4 \varepsilon_{0} \varepsilon_{a} U_{0}^{2}}{\gamma_{1} d}\left(\frac{\sqrt{2}}{\pi} \frac{U}{U_{0}} \frac{1}{\ln \left(\frac{d}{\xi} \frac{U_{0}}{U}\right)}-2 \gamma\right),
$$

which yields the velocity-voltage coefficient $k=d v / d U$ :

$$
k=\frac{4 \sqrt{2} \varepsilon_{0} \varepsilon_{a} U_{0}}{\pi \gamma_{1}} \frac{1}{d} \frac{1}{\ln \left(\frac{d}{\xi} \frac{U_{0}}{U}\right)}\left(1+\frac{1}{\ln \left(\frac{d}{\xi} \frac{U_{0}}{U}\right)}\right) .
$$

We see that $k$ decreases roughly as $1 / d$, whereas it is only weakly dependent on $U$ (recall that $d / \xi$ is large), which explains the linearity of the dependence in Fig. 3. On a closer inspection, there one can detect a slightly increasing slope for higher voltages. Equation (27) also predicts this effect, which comes from the decreasing volume of dissipation.

Using the result (27) we fitted the simulated values of the velocity-voltage coefficient $k$ by a fitting function of the form

$$
k=\frac{a_{1}}{d} \frac{1}{\ln \left(\frac{d}{a_{2}} \frac{U_{0}}{U_{e q}}\right)}\left(1+\frac{1}{\ln \left(\frac{d}{a_{2}} \frac{U_{0}}{U_{e q}}\right)}\right),
$$

where $a_{1}$ and $a_{2}$ are the fitting parameters. The fit is presented in Fig. 11.

\section{Front velocity near the saddle node}

Let us concentrate now on the nonlinearity of the velocity-voltage dependence in Fig. 3. For $U_{\min }<U<U_{\max }$ the system exhibits bistability, i.e., both the wall and the disclination configurations are (meta)stable, and one has front solutions connecting the two states. We will present a description of the front velocity valid near the lower saddle node (near $U_{\text {min }}$ ) using the simplest generic one-dimensional Ginzburg-Landau model for an effective scalar order parameter $u$, completely neglecting the structure in the $x z$ plane. A suitable choice of the order parameter $u$ is the $\mathrm{Q}_{z z}$ component of the nematic $Q$ tensor in the middle of the wall, which measures the degree of ordering with respect to the $z$ axis. In the middle of the Fréedericksz wall the director is parallel to 
the $x$ axis, hence $\mathrm{Q}_{z z}<0$. It is a monotonically increasing function of the voltage. In the disclination state the director in the middle of the pinched wall is parallel to the $z$ axis, hence $Q_{z z}>0$. In order to simplify the description we choose $u=\mathrm{Q}_{z z}-\mathrm{Q}_{z z}^{\text {wall }}\left(U_{\min }\right)$, where $\mathrm{Q}_{z z}^{\text {wall }}\left(U_{\min }\right)$ is the value of $\mathrm{Q}_{z z}$ in the middle of the wall at $U_{\text {min }}$.

The transition can now be described in terms of a control parameter $\epsilon \propto U^{2}-U_{\min }^{2}$ by the equation

$$
\tau \partial_{t} u=\xi^{2} \partial_{y}^{2} u+f(u), \quad f(u)=-u(u-a)^{2}+\epsilon u,
$$

where $\tau$ and $\xi$ are the analogs of the characteristic time scale and correlation length introduced earlier. The dependence of the dissipation (determining the left-hand side of Eq. (29)) on the control parameter is ignored as it is not expected to exhibit any pecularities at the bifurcation. We can rewrite

$$
f(u)=-u\left(u-u_{s}\right)\left(u-u_{1}\right), \quad u_{1, s}=a \pm \epsilon^{1 / 2},
$$

which shows that we are indeed dealing with a saddle-node bifurcation. The stable solution $u_{1}$ emerging for $\epsilon>0$ represents the disclination state, while the solution $u=0$ represents the wall state. The propagating front solutions $u(y-v t)$ for this model can be found analytically. The front connecting the states $u=0$ and $u=u_{1}$ is captured by the ansatz $\partial_{y} u$ $=\alpha u\left(u-u_{1}\right)$ which solves the equation. One then finds $\alpha$ $=1 /(\sqrt{2} \xi)$ and

$$
|v|=\frac{\xi}{\sqrt{2} \tau}\left(u_{1}-2 u_{s}\right)=\frac{\xi}{\sqrt{2} \tau}\left(-a+3 \epsilon^{1 / 2}\right) .
$$

Thus, near the saddle node the experimentally observed velocity should behave like

$$
|v| \propto-a+b\left(U^{2}-U_{\min }^{2}\right)^{1 / 2},
$$

which we indeed observe as shown in the inset of Fig. 3.

\section{CONCLUSIONS}

We have presented an experimental and theoretical study of the pincement phenomenon-transformation of a Fréedericksz wall into a pair of disclination lines. Experimentally we studied the dynamics of the boundary (front) between the Fréedericksz wall and the disclination structure and measured the front velocity as a function of the voltage. We also observed the transformation of a planar wall into a twisted wall and determined the threshold of this transition. The experimental results were compared to the results of numerical simulations based on the nematic tensor order parameter. The motion of the front and the correct velocity dependence were recovered in the simulation, whereas an exact quantitative comparison of the dynamics is difficult as a large system is required in the simulation due to the presence of defects. By introducing reduced static and dynamic models we obtained approximate expressions for the equlibrium voltage and front velocity, which were used to extrapolate the results of the simulations to the cell thickness used in the experiment. We uncovered a bifurcation scenario incorporating a pair of saddle nodes at which the wall and disclination solutions appear or disappear, which was done numerically by calculating the behavior of a suitably chosen order parameter in the vicinity of the saddle nodes. The nonlinear behavior of the front velocity near the lower saddle node is then explained by the bifurcation scenario.

\section{ACKNOWLEDGMENTS}

A.deL. has been supported by a grant from the Deutsche Forschungsgemeinschaft (Grant No. GRK 698). D.S. gratefully acknowledges the financial support of the Slovenian Office of Science (Grant No. P1-0099) and the Alexander von Humboldt Foundation.
[1] T. W. B. Kibble, J. Phys. A 9, 1387 (1976).

[2] W. H. Zurek, Nature (London) 317, 505 (1985).

[3] I. Chuang, R. Durrer, N. Turok, and B. Yurke, Science 251, 1336 (1991).

[4] K. Minoura, Y. Kimura, K. Ito, R. Hayakawa, and T. Miura Phys. Rev. E 58, 643 (1998).

[5] G. Tóth, C. Denniston, and J. M. Yeomans, Phys. Rev. Lett. 88, 105504 (2002).

[6] C. Williams, P. Pieranski, and P. E. Cladis, Phys. Rev. Lett. 29, 90 (1972).

[7] L. Léger, Solid State Commun. 10, 697 (1972).

[8] L. Léger, Mol. Cryst. Liq. Cryst. 24, 33 (1973).

[9] F. Brochard, J. Phys. (Paris) 33, 607 (1972).

[10] W. H. de Jeu, C. J. Gerritsma, and T. W. Lathouwers, Chem. Phys. Lett. 14, 503 (1972).

[11] P. E. Cladis, W. van Saarloos, P. L. Finn, and A. R. Kortan, Phys. Rev. Lett. 58, 222 (1987).

[12] A. Bogi, P. Martinot-Lagarde, I. Dozov, and M. Nobili, Phys. Rev. Lett. 89, 225501 (2002).

[13] N. Schopohl and T. J. Sluckin, Phys. Rev. Lett. 59, 2582
(1987).

[14] D. Svenšek and S. Žumer, Phys. Rev. E 66, 021712 (2002).

[15] P. G. de Gennes and J. Prost, The Physics of Liquid Crystals (Clarendon Press, Oxford, 1993).

[16] A. Stieb, G. Baur, and G. Meier, J. Phys. (Paris), Colloq. 36, C1-185 (1975).

[17] C. Chevallard, M. Nobili, and J.-M. Gilli, Liq. Cryst. 28, 179 (2001).

[18] K. S. Krishnamurthy and R. Balakrishnan, Liq. Cryst. 29, 383 (2002).

[19] I. Rehberg, B. L. Winkler, M. de la Torre Juárez, S. Rasenat, and W. Schöpf, Adv. Solid State Phys. 29, 35 (1989).

[20] T. M. Bock, J. Bläsing, V. Frette, and I. Rehberg, Rev. Sci. Instrum. 71, 2800 (2000).

[21] S. Rasenat, G. Hartung, B. L. Winkler, and I. Rehberg, Exp. Fluids 7, 412 (1989).

[22] L. Bata, A. Buka, and I. Janossy, Solid State Commun. 15, 647 (1974).

[23] L. M. Blinov, Electro-Optical and Magneto-Optical Properties of Liquid Crystals (Wiley, New York, 1983). 
[24] E. F. Carr, Mol. Cryst. Liq. Cryst. 7, 253 (1969).

[25] W. Helfrich, J. Chem. Phys. 51, 4092 (1969).

[26] A. de Lózar, T. Bock, M. Müller, W. Schöpf, and I. Rehberg, New J. Phys. 5, 63 (2003).

[27] E. Bodenschatz, W. Zimmermann, and L. Kramer, J. Phys. (Paris) 49, 1875 (1988).

[28] B. R. Ratna and R. Shashidhar, Mol. Cryst. Liq. Cryst. 42, 113 (1977).

[29] G. P. Chen, H. Takezoe, and A. Fukuda, Liq. Cryst. 5, 341 (1989).

[30] H. Herba, A. Szymanski, and A. Drzymala, Mol. Cryst. Liq.
Cryst. 127, 153 (1985).

[31] C. Chevallard, M. Clerc, P. Coullet, and J.-M. Gilli, Eur. Phys. J. E 1, 179 (2000).

[32] T. Nagaya and J.-M. Gilli, Phys. Rev. E 65, 051708 (2002).

[33] T. Qian and P. Sheng, Phys. Rev. E 58, 7475 (1998).

[34] H. J. Coles, Mol. Cryst. Liq. Cryst. 49, 67 (1978).

[35] M. L. Magnuson, B. M. Fung, and J. P. Bayle, Liq. Cryst. 19, 823 (1995).

[36] H. Imura and K. Okano, Phys. Lett. 42A, 403 (1973).

[37] G. Ryskin and M. Kremenetsky, Phys. Rev. Lett. 67, 1574 (1991). 